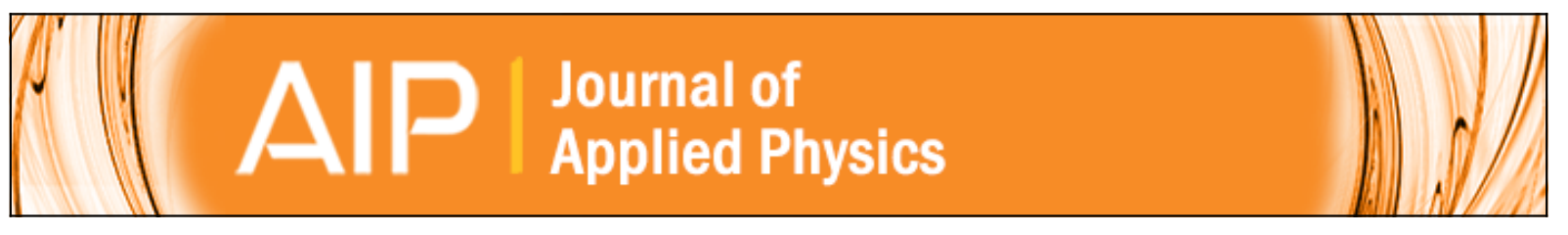

Magnetoresistive effects in perpendicularly magnetized Tb-Co alloy based thin films and spin valves

M. Gottwald, M. Hehn, F. Montaigne, D. Lacour, G. Lengaigne, S. Suire, and S. Mangin

Citation: Journal of Applied Physics 111, 083904 (2012); doi: 10.1063/1.3703666

View online: http://dx.doi.org/10.1063/1.3703666

View Table of Contents: http://scitation.aip.org/content/aip/journal/jap/111/8?ver=pdfcov

Published by the AIP Publishing

Articles you may be interested in

Light-induced magnetization reversal of high-anisotropy TbCo alloy films

Appl. Phys. Lett. 101, 162408 (2012); 10.1063/1.4759109

Enhanced difference in switching fields for perpendicular magnetic spin valves with a composite [ Co / $\mathrm{Ni}$ ] $\mathrm{N} /$

TbCo reference layer

J. Appl. Phys. 109, 023907 (2011); 10.1063/1.3536476

Effect of crystalline orientation and lattice distortion of FeCo spin polarization enhancement layer in perpendicular magnetic tunnel junction with RE-TM alloy films

J. Appl. Phys. 107, 09C719 (2010); 10.1063/1.3358601

Influence of spin polarization enhancement layer to rare earth-transition metal thin films for perpendicular tunneling magnetoresistance evaluated by ferromagnetic Hall effect

J. Appl. Phys. 99, 08 C513 (2006); 10.1063/1.2177420

Magnetoresistance in amorphous TbFeCo films with perpendicular magnetic anisotropy

J. Appl. Phys. 97, 10C515 (2005); 10.1063/1.1853911

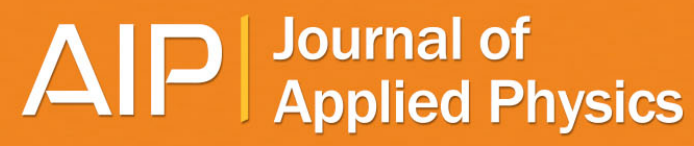

Journal of Applied Physics is pleased to announce André Anders as its new Editor-in-Chief 


\title{
Magnetoresistive effects in perpendicularly magnetized Tb-Co alloy based thin films and spin valves
}

\author{
M. Gottwald, M. Hehn, F. Montaigne, D. Lacour, G. Lengaigne, S. Suire, and S. Mangin \\ Institut Jean Lamour, CNRS UMR 7198, Nancy-Université, B.P. 239 F-54506, Vandoeuvre lès Nancy, France
}

(Received 13 January 2012; accepted 10 March 2012; published online 16 April 2012)

\begin{abstract}
$\mathrm{Tb}$-Co ferrimagnetic alloy thin films and spin valves have been grown to study their magnetoresistance response in various geometries. The studied $\mathrm{Tb}$-Co alloys show strong perpendicular anisotropy and tunable magnetization by several orders of magnitude. Magnetoresistance signals such as giant magnetoresistance (GMR), anisotropic magnetoresistance (AMR), extraordinary Hall effect (EHE), and magnon magnetoresistance (MMR) have been studied. The angular dependence of those magnetoresistive effects is also investigated. Finally we demonstrate that by adjusting the Tb-Co layer composition in a spin valve structure, the sign and the amplitude of the GMR and EHE signal can be tuned. (C 2012 American Institute of Physics. [http://dx.doi.org/10.1063/1.3703666]
\end{abstract}

\section{INTRODUCTION}

Spin valve structures composed of two magnetic layers separated by a nonmagnetic layer with perpendicular anisotropy have attracted much interest recently because of their potential application for spintronic memory devices. ${ }^{1}$ Spinpolarized current could be used to reverse the magnetization orientation in nanopillar spin valves to form spin transfer torque magnetic random access memory (STT-MRAM) devices. $^{2}$ For these applications devices with perpendicular magnetic anisotropy ${ }^{3-6}$ have shown to reduce the threshold current needed to provide magnetization switching. In those systems, the magnetization reversal is directly related to the thermal stability of the free layers. ${ }^{4}$ To further reduce the switching current without affecting the thermal stability, critical for applications, "novel" materials or/and structures need to be grown. Since spin transfer torque switching is based on angular momentum conservation, one way to decrease the amount of spin transfer needed to reverse the free layer is to decrease its magnetization. ${ }^{7}$ Another advantage of using low magnetization material for data storage is its low coupling to any external magnetic field that could erase stored information. Using these low moment materials would be a route to increasing density in bit patterned media data storage $^{8}$ or MRAM without increasing dipolar field interactions. ${ }^{9}$ In this context, rare earth - transition metals ferromagnetic materials are very interesting since they exhibit a strong anisotropy due to their anisotropic $4 f$ orbitals. In the case of heavy rare earths, their magnetization may be tuned because the magnetization of the heavy rare earth sublattice is antiferromagnetically coupled to the magnetization of the transition metal sublattice. Because magnetic moment of these materials is low, the study of their magnetic properties using conventional magnetometry is difficult. In this case, magnetotransport measurements may be helpful to investigate magnetization reversal in such systems.

This paper focuses on ferrimagnetic Tb-Co alloys showing strong perpendicular anisotropy and tunable magnetization. The intrinsic properties of single thin films are shown and both temperature and composition dependence are presented. Magnetotransport measurements of single thin
Tb-Co films allows for the isolation of different contributions to the magnetoresistance. Finally, Tb-Co based spin valves are studied. Magnetization, giant magnetoresistance (GMR), anisotropic magnetoresistance (AMR), extraordinary Hall resistance (EHE), and magnon magnetoresistance (MMR) are studied in a current in plane geometry. The sign of the EHE and GMR magnetoresistance signals is found to depend strongly on the Tb-Co composition.

\section{SAMPLES}

Tb-Co alloys and $\mathrm{Tb}$-Co based spin valves were grown by DC magnetron sputtering with a base pressure less than $5 \times 10^{-9}$ mbar. Pure $\mathrm{Co}$ and $\mathrm{Tb}$ targets were used for a cosputtering process and the relative atomic concentration of the two elements $\mathrm{Co}$ and $\mathrm{Tb}$ was controlled by the relative sputtering power. Samples were deposited on Si (100) substrates covered first with a $5 \mathrm{~nm}$ thick Ta seed layer. The multilayer is protected from oxidation by a $\mathrm{Cu} / \mathrm{Pt}$ bilayer.

\section{MAGNETORESISTIVE EFFECTS IN SINGLE Tb-Co THIN FILMS}

Tb-Co alloys are sperimagnetic ${ }^{10}$ and their magnetic characteristics have been described in detail by Hansen et al. ${ }^{11}$ The average magnetization held by the $\mathrm{Tb}$ sublattice is coupled antiparallel to magnetization of the Co one. For a given temperature, the net magnetization is then found to be zero for a composition called the compensation composition. The temperature for which the net magnetization is zero (called the compensation temperature) is found to be dependent on composition since the magnetization of the Tb sublattice depends strongly on temperature. As shown in Fig. 1, those two behaviors were clearly observed on the $\mathrm{Si} / \mathrm{SiO}(100$ $\mathrm{nm}) / \mathrm{Ta}(5 \mathrm{~nm}) / \mathrm{Tb}_{X} \mathrm{Co}_{1-X}(20 \mathrm{~nm}) / \mathrm{Cu}(2 \mathrm{~m}) / \mathrm{Pt}(2 \mathrm{~nm})$ layers. At room temperature, a compensation composition of $x_{\text {comp }}$ $=0.79$ was determined Fig. 1(a). Above this composition, the alloy is called Co dominant since the magnetization of the Co sublattice is larger than the Tb sublattice magnetization. On the other hand, for a larger amount of $\mathrm{Tb}$ the alloy is called $\mathrm{Tb}$ dominant and the net magnetization is parallel to 

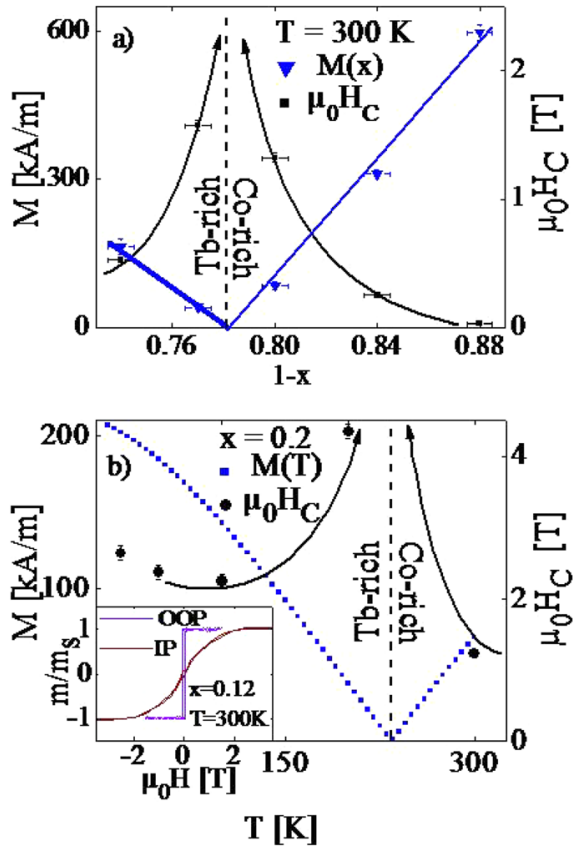

FIG. 1. Magnetization $\mathrm{M}$ and coercivity $\mu_{0} H_{C}$ of $\mathrm{Co}_{1-x} \mathrm{~Tb}_{x}(20 \mathrm{~nm})$ alloys obtained from SQUID magnetometry: (a) $M$ and $\mu_{0} H_{C}$ as a function of the Co concentration 1- $x$ at room temperature. (b) $M$ and $\mu_{0} H_{C}$ as a function of temperature for a $\mathrm{Co}_{80} \mathrm{~Tb}_{20}$ alloy. Inset: In-plane (IP) and out-of-plane (OOP) hysteresis loop for a $\mathrm{Co}_{88} \mathrm{~Tb}_{12}$ alloy at room temperature. Lines are guides to the eye.

the average magnetization of the Tb sublattice. For a given composition of $x=0.8$, shown in Fig. 1(b), the magnetization decreases as the temperature decreases starting from room temperature and crosses zero for a compensation temperature of $T_{\text {comp }}=235 \mathrm{~K}$. Note that for those compositions around the magnetization compensation, all the samples exhibit a strong perpendicular magnetic anisotropy shown by a square hysteresis loop for an applied field perpendicular to the film plane. The evolution of the coercivity versus composition shows that as the magnetization of the $\mathrm{Tb}$-Co layer decreases, the coercivity increases and diverges when the magnetization tends to zero. The above description underlines the fact that in the Tb-Co system, magnetization and coercivity may easily be tuned by adjusting the composition and/or the temperature. The next step consists of investigating the magnetoresistance response of this class of materials to determine their potential spintronic applications.

In order to study the different magnetoresistance effects, thin Tb-Co films have been patterned as shown in the inset of Fig. 2(d). This type of geometry allows for performing four-point-probe measurements in a current in plane (CIP) geometry as well as Hall resistance measurements in any applied field direction. Figure 2 shows measurements on $\mathrm{Si} /$ $\mathrm{SiO}(100 \mathrm{~nm}) / \mathrm{Ta}(5 \mathrm{~nm}) / \mathrm{Co}_{88} \mathrm{~Tb}_{12}(20 \mathrm{~nm}) / \mathrm{Cu}(2 \mathrm{~nm}) / \mathrm{Pt}(2 \mathrm{~nm})$ with a field applied along different orientations. For this composition, the Tb-Co magnetization is parallel to the contribution of the Co sublattice.

Let us first concentrate on the results obtained when the field is applied along the film easy axis (perpendicular to the film plane $\phi=0$ ). The observed square loop with a sharp reversal is due to the perpendicular anisotropy and the fact that this method probes only the small volume inside the Hall
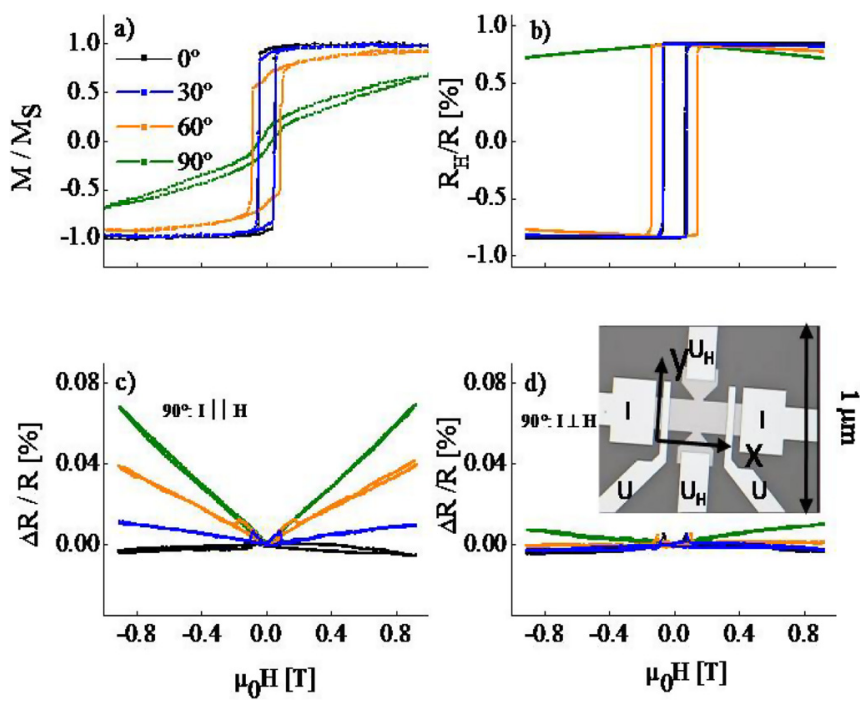

FIG. 2. Angular dependence of the magnetoresistance of $\mathrm{C} \mathrm{Co}_{88} \mathrm{~Tb}_{12}(20 \mathrm{~nm})$ film: (a) VSM hysteresis loops as a function of the angle between the perpendicular easy axis and the applied field. (b) Hall resistance. (c) Four- pointprobe current in-plane (CIP) resistance (an angle of $90^{\circ}$ corresponds to the case where current and field are parallel, $\mathrm{x}$ direction). (d) CIP resistance (for an angle of $90^{\circ}$ current and field are perpendicular, $y$ direction). Inset: Optically patterned Hall cross $(200 \times 800 \mu \mathrm{m})$ for CIP measurements. The absolute four-point-probe CIP resistance of the samples is $\sim 161 \Omega$. The distance between the two electrodes denoted with $U$ is $400 \mu \mathrm{m}$.

cross. A large extraordinary Hall signal with a low signal-tonoise ratio is measured as shown in Fig. 2(b). This is consistent with the presence of heavy rare earth atoms for which the spin-orbit coupling is large. ${ }^{12}$ As illustrated in the next section and reported in reference, ${ }^{13}$ we observed that the Hall resistivity is negative for $\mathrm{Tb}$ rich compositions and positive for Co rich alloys.

Unlike the EHE measurement the four-point-probe signal in Fig. 2 is extremely weak with a relative magnetoresistance maximum of about $0.004 \%$ for a four-point-probe resistance of about $162 \Omega$. The peaks observed at the reversal field may be due to the presence of domain walls. The existence of domain walls may give rise to an AMR signal since the resistivity is given by $\rho(\theta)=\rho_{\perp}+\Delta \rho \cos ^{2}(\theta)$ where $\Delta \rho=\rho_{/ /}-\rho_{\perp}$ with $\rho_{/ /}$the resistivity when the magnetization $(M)$ is parallel to the applied current I and $\rho_{\perp}$ the resistivity when $M$ is perpendicular to $I$ and $\theta$ is the angle between $M$ and $I .{ }^{14}$ It could also be due to more complex domain wall magnetoresistance as described in several references. ${ }^{15,16}$ Apart from the peak, a linear decrease of the resistance with the applied field may be observed. The magnitude of the signal is very weak and two origins are possible. It could be due to an AMR signal coming from a decrease of the sperimagnetic cone angle of $\mathrm{Tb}$ with increasing applied perpendicular field and/or consistent with the presence of electron-magnon diffusion leading to a linear magnon magnetoresistance variation as observed for materials with strong anisotropy like in Fe-Pt. ${ }^{17}$ However, it is a very weak signal in the case of $\mathrm{Tb}-\mathrm{Co}$ and cannot provide a reliable way to detect magnetization reversal.

On Fig. 2 the influence of the orientation of the applied field on the magnetoresistance response is shown as the field is tilted away from the perpendicular to an in-plane direction. 
Two types of field tilting are considered: The field may be tilted toward the current direction $(x)$ or toward the perpendicular to the current direction (y) as defined in Fig. 2(d).

No influence on the tilting direction is observed for the EHE. Since the EHE resistance is proportional to the component perpendicular to the film plane, the hall resistance decreases when the field is applied in plane. The weak reversible decrease that can be observed (less than $15 \%$ as 0.8 $\mathrm{T}$ is applied in plane) proves that the anisotropy field for such alloys is very large. This is due to their weak magnetization the presence of very anisotropic $4 f \mathrm{~Tb}$ atoms. The anisotropy field $H_{K}$ given by $H_{K}=K / 2 M_{S}$ where $K$ is the anisotropy constant and $M_{S}$ is the saturation magnetization, and can be evaluated from magnetometry to about $3 \mathrm{~T}$ for this $\mathrm{Co}_{0.88} \mathrm{~Tb}_{0.12}$ alloy.

For the magnetoresistance measured with the four-pointprobe geometry the first observation is that the way the field is tilted, strongly influences the amplitude of the magnetoresistance signal. In both cases, the slope increased as the angle between the out of plane direction and the applied field was increased. However, the amplitude is much bigger as the magnetization is tilted along the field direction. This is consistent with the explanation that the signal comes from an AMR phenomena and that the resistance is larger when the current $(I)$ is parallel to the magnetization $(M)$. The weak signal measured as the field is tilted in the plane perpendicular to I may be due to domain formation as described by Ref. 14 .

For single Tb-Co thin films, measuring EHE is clearly the most accurate way to probe its magnetization orientation.

\section{MAGNETORESISTIVE EFFECTS IN Tb-Co BASED SPIN VALVE STRUCTURES}

From the above information on the magnetic properties of the single ferromagnetic layer we have grown three types of spin valve structures (thicknesses in $\mathrm{nm}$, concentrations in atomic percent) $\left(\mathrm{Tb}_{12} \mathrm{Co}_{88}(20) / \mathrm{Cu}(3.5) / \mathrm{Tb}_{20} \mathrm{Co}_{80}(20)\right.$ (Co dominant alloy/Cu/Co dominant alloy), $\mathrm{Tb}_{12} \mathrm{Co}_{88}(20) / \mathrm{Cu}(3.5) /$ $\mathrm{Tb}_{26} \mathrm{Co}_{74}(20)$ (Co dominant alloy/Cu/Tb dominant alloy) and
$\mathrm{Tb}_{23} \mathrm{Co}_{77}(20) / \mathrm{Cu}(3.5) / \mathrm{Tb}_{26} \mathrm{Co}_{74}(20)$ ( $\mathrm{Tb}$ dominant alloy/Cu/Tb dominant alloy). Those structures have then been patterned using optical lithography to create devices as presented on the inset of Fig. 2. Two types of magnetoresistance measurements as well as the magnetization measurements performed before lithography are shown on Fig. 3 for the three samples described above. The giant magnetoresistance effect can explain the observed signal as shown in similar in-plane devices. ${ }^{18-21}$ The amplitude is typically $0.02 \%$. This small value is explained by spin-orbit scattering in the presence of $4 f$ atoms $^{18}$ and shunting through the thick layer stacking. All the magnetoresistance measurements may be explained by considering the relative angle between the Co sublattices of the two Tb-Co layers $\Delta R$ $=\Delta R \cos (\theta)$ where $\theta$ is the angle between the two Co sublattices. When the two Co sublattices are parallel, the resistance is minimum. The antiparallel configuration has maximum resistance. Positive and negative GMR effects may then be observed depending on the concentration of the two alloys.

Furthermore, we can observe that the extraordinary Hall coefficient of the $\mathrm{Tb}$-Co layers can be either positive or negative depending on the Tb-Co composition. Similar to Ref. 13 we observe a positive Hall coefficient for Co rich alloys and a negative Hall coefficient for Tb rich alloys. It is still unclear if the Hall coefficient in rare earth - transition metal alloys is due to the rare earth sublattice (with a positive Hall coefficient), or the transition metal sublattice (with a negative Hall coefficient), or due to both. ${ }^{12}$ Each of these models would explain the observed behavior.

\section{EFFECT OF THE FIELD ANGLE ON MAGNETORESISTANCE}

SQUID magnetometry, EHE and GMR measurements are complementary techniques since the SQUID gives information on the component of the magnetization parallel to the field, the GMR signal measures the relative orientation of the Co sublattices of the two alloys and the Hall effect depends on the component of the magnetization which is perpendicular to the film plane.
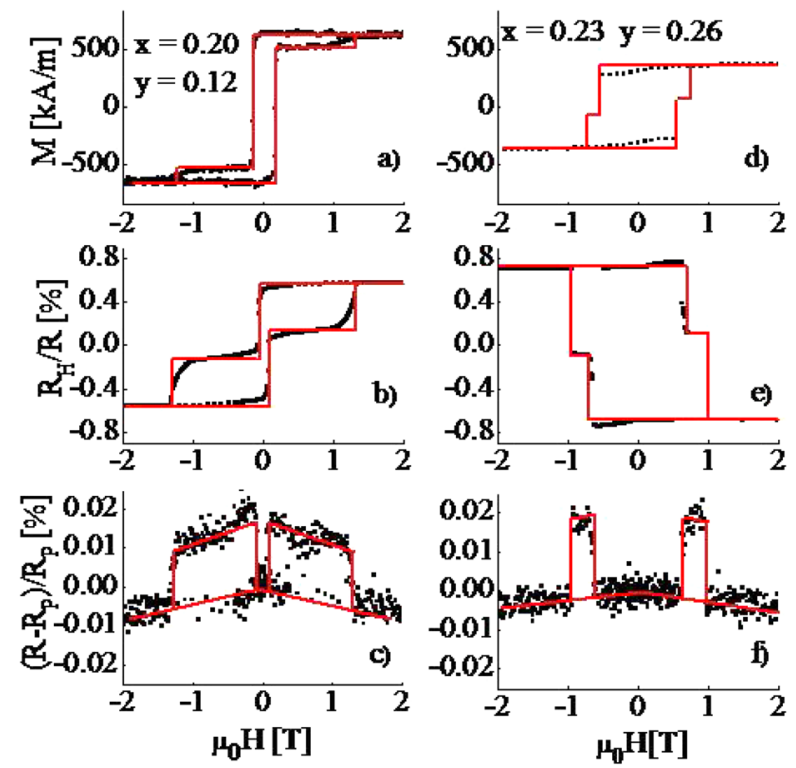

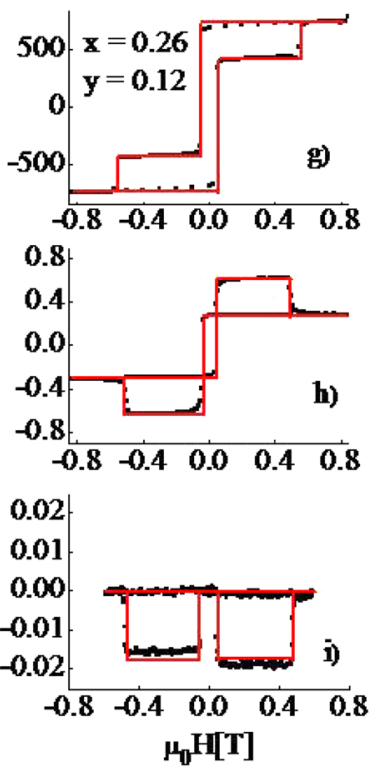

FIG. 3. SQUID hysteresis loops (a,d,g), Hall resistance (b,e,h) and four-point-probe CIP resistance $(\mathrm{c}, \mathrm{f}, \mathrm{i})$ for a series of $\mathrm{Co}_{1-\mathrm{y}} \mathrm{Tb}_{\mathrm{y}}(20 \mathrm{~nm}) /$ $\mathrm{Cu}(3.5 \mathrm{~nm}) / \mathrm{Co}_{1-x} \mathrm{~Tb}_{x}$ spin valves: (a)-(c) $x$ $=0.20$ and $y=0.12 ;(\mathrm{d})-(\mathrm{f}) x=0.23$ and $y$ $=0.26$; (g) - (i) $x=0.26$ and $y=0.12$. Lines are guides to the eye. 

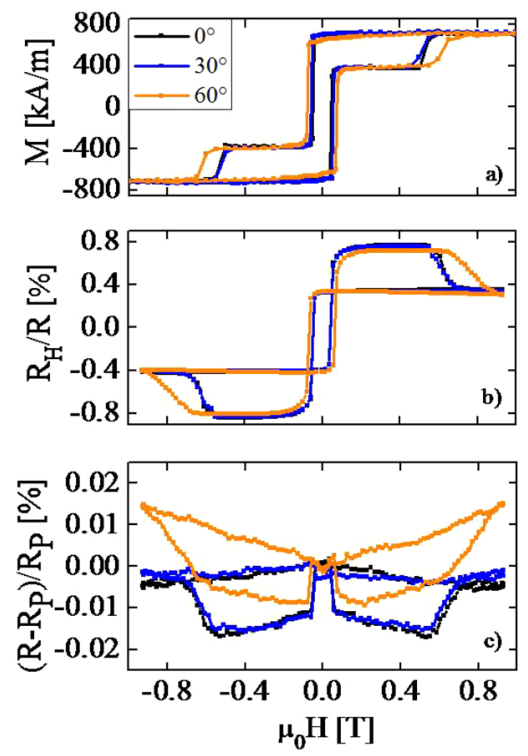

FIG. 4. Dependence of the magnetoresistance of a $\mathrm{Co}_{88} \mathrm{~Tb}_{12}(20 \mathrm{~nm}) /$ $\mathrm{Cu}(3.5) / \mathrm{Co}_{74} \mathrm{~Tb}_{26}(20 \mathrm{~nm})$ spin valve on the angle between the applied field and the perpendicular easy axis. (a) VSM hysteresis loops and (b) Hall resistance. (c) Four-point-probe CIP resistance (the field is always perpendicular to the current).

In order to illustrate this phenomenon, we present on Fig. 4 the influence of the applied field orientation on the three types of measurements for the $\mathrm{Tb}_{12} \mathrm{Co}_{88}(20) / \mathrm{Cu}(3.5) / \mathrm{Tb}_{26} \mathrm{Co}_{74}(20)$ (Co dominant alloy/ $\mathrm{Cu} / \mathrm{Tb}$ dominant alloy) spin valve. The coercivity of both $\mathrm{Co}-\mathrm{Tb}$ layers increases with increasing angle from the easy perpendicular axis as the Zeeman energy for the mostly perpendicular oriented magnetization becomes less important. The maxima for the amplitudes of the Hall resistance are obtained at zero field. With increasing field, the amplitudes of the Hall resistance decrease because the absolute value of the $\mathrm{z}$ component of the magnetization shrinks with increasing in-plane fields. For the four-point-probe resistance, we observe a change of the slope of the resistance with field when the angle between field and perpendicular easy axis is increased. For small angles, the resistance decreases with increasing field due to magnon magnetoresistance as explained above for the case of a single Tb-Co layer. For larger angles the resistance increases with increasing field similar to the case of the single Tb-Co layer, and is evidence of domain formation. Since the applied fields of up to $0.9 \mathrm{~T}$ are small compared to the anisotropy fields of both layers $\left(3 \mathrm{~T}\right.$ for $\mathrm{Tb}_{12} \mathrm{Co}_{88}$ and $5.5 \mathrm{~T}$ for $\mathrm{Tb}_{26} \mathrm{Co}_{74}$ ), only a small misalignment between the magnetic moments can be expected. Thus a possible decrease of the amplitude of the GMR effect is too small to be measured due to the already small GMR effect in this material.

\section{CONCLUSION}

In conclusion, we have grown single $\mathrm{Tb}$-Co alloy thin films and Tb-Co based spin valve structures and studied the magnetoresistance signals depending on the current geometry, the Tb-Co concentration and the field orientation. Three types of effects have clearly been identified: anisotropic magnetoresistance, giant magnetoresistance, and extraordinary Hall effect. The amplitudes of the first two effects are small, around $0.02 \%$ for GMR and $0.08 \%$ for AMR. In contrast, EHE generates a strong signal which is very useful for observing magnetization reversal. The influence of the alloy concentration on the EHE and the GMR was studied. The sign of the EHE is positive for Co rich alloys and negative for $\mathrm{Tb}$ dominant alloys, whereas the sign of the GMR depends on the relative concentrations. Same dominance leads to a positive GMR effect, opposite dominance leads to a negative GMR effect.

Tb-Co alloys thus possess several properties which can be used as tools to precisely observe magnetization reversal, especially in nano objects where detection by classical magnetometry is difficult.

\section{ACKNOWLEDGMENTS}

Authors thank J. J. Kan from CMRR, San Diego, CA for proofreading of the manuscript.

${ }^{1}$ W. J. Gallagher and S. S. P. Parkin, IBM J. Res. Dev. 50, 5 (2006).

${ }^{2}$ J. A. Katine and E. E. Fullerton, J. Magn. Magn. Mater. 320, 1217 (2008).

${ }^{3}$ S. Mangin, D. Ravelosona, J. A. Katine, M. J. Carey, B. D. Terris, and E. E. Fullerton, Nature Mater. 5, 210 (2006).

${ }^{4}$ S. Mangin, Y. Henry, D. Ravelosona, J. A. Katine, and E. E. Fullerton, Appl. Phys. Lett. 94, 012502 (2009).

${ }^{5}$ H. Meng and J. P. Wang, Appl. Phys. Lett. 88, 172506 (2006).

${ }^{6}$ T. Seki, S. Mitani, K. Yakushiji, and K. Takanashi, Appl. Phys. Lett. 88, 172504 (2006).

${ }^{7}$ J. Sun, IBM J. Res. Dev. 50(1), 81-100 (2006).

${ }^{8}$ B. D. Terris and T. Thomson, J. Phys. D: Appl. Phys. 38, R199-R222 (2005).

${ }^{9}$ T. Devolder, C. Maufront, J.-V. Kim, H.W. Schumacher, C. Chappert, and R. Fournel, IEEE Proc.-Sci. Meas. Technol. 152(4), 196-200 (2005).

${ }^{10}$ J. J. Rhyne, Amorphous Magnetic Rare Earth Alloys (North-Holland, Amsterdam, 1979).

${ }^{11}$ P. Hansen et al., J. Appl. Phys. 66, 756 (1989).

${ }^{12}$ J. Moritz, B. Rodmacq, S. Auffret, and B. Dieny, J. Phys. D: Appl. Phys. 41, 135001 (2008).

${ }^{13}$ T. W. Kim and R. J. Gambino, J. Appl. Phys. 87, 1869 (2000).

${ }^{14}$ R. A. Hajjar, M. Mansuripur, and H.-P. D. Shieh, J. Appl. Phys. 69, 7067 (1991).

${ }^{15}$ M. Viret, D. Vignoles, D. Cole, W. Allen, D. S. Daniel, and J. F. Gregg, Phys. Rev. B 53, 8464 (1996).

${ }^{16}$ G. Tatara and H. Fukayama, Phys. Rev. Lett. 78, 3773 (1997).

${ }^{17}$ A. P. Mihai, J. P. Attané, A. Marty, P. Warin, and Y. Samson, Phys. Rev. B 77, 060401 (2008).

${ }^{18}$ C. Bellouard, H. D. Rapp, B. George, S. Mangin, and G. Marchal, Phys. Rev. B 53, 5082 (1996).

${ }^{19}$ T. Hauet, F. Montaigne, M. Hehn, Y. Henry, and S. Mangin, Appl. Phys. Lett. 93, 222503 (2008).

${ }^{20}$ X. J. Bai, J. Du, J. Zhang, B. You, L. Sun, W. Zhang, X. S. Wu, S. L. Tang, A. Hu, H. N. Hu, and S. M. Zhou, J. Appl. Phys. 103, 07 F305 (2008).

${ }^{21}$ C. Kaiser, A. F. Panchula, and S. S. P. Parkin, Phys. Rev. Lett. 95, 047202 (2005). 